\title{
Frente a los desafíos actuales de la geopolítica global, ¿cómo se articula la autonomía estratégica de la Unión Europea en el marco de su política exterior y de seguridad?
}

\author{
Miguel Ángel Benedicto Solsona \\ Y María José Molina García*
}

* Miguel Ángel SOLSONA,

Doctor en Relaciones Internacionales, Profesor adjunto en Universidad Europea de Madrid y Profesor Asociado en Universidad Complutense de Madrid. Es miembro del Consejo del Movimiento Europeo Internacional y copresidente del Itinerario Cultural Europeo Vía

Carlomagno.

María José MOLINA GARCÍA,

Profesora acreditada contratada

Doctora (ANECA). Responsable de Programas en Centro de Estudios Garrigues. Doctora en Ciencias

Políticas y de la Administración de la UCM. Experiencia docente e investigadora en Centros universitarios públicos y privados. Participación en Comités y otras representaciones. Evaluadora externa en diversas Revistas.

Recibido:

18/11/2019

Aceptado: 08/05/2020

https://doi.org// 0.15366/relacionesinternacionales2020.44.00I

Formato de citación recomendado:

BENEDICTO SOLSONA, Miguel Ángel y MOLINA GARCÍA, María José (2020), "Frente a los desafíos actuales de la geopolítica global, ¿cómo se articula la autonomía estratégica de la Unión Europea en el marco de su política exterior y de seguridad?", Relaciones Internacionales, $n^{\circ} 44$, pp. I I-28. 


\title{
TITLE
}

Facing the current challenges of global geopolitics, how is the strategic autonomy of the European Union articulated in the framework of its foreign and security policy?

\begin{abstract}
The changing global geopolitical circumstances (with a revisionist Russia, a rising and more assertive China, a withdrawing United States with values increasingly different from those of Europe, the instability coming from the East and the Southern Mediterranean, the consummation of Brexit...) are the contextual backdrop of the European Union, and following the adoption of its Global Strategy on Foreign and Security Policy it has developed a series of political and institutional tools to support its strategic autonomy in the field of security and defence (Action Plans, EU-NATO cooperation, permanent structured cooperation, the European Initiative for Intervention...). To these instruments can be added others provided for in the Lisbon Treaty (2009), such as the solidarity and mutual assistance clauses, the latter invoked after the Daesh attack in Paris in November 2015, as a defensive alliance to guarantee security in European countries. All this is in line with the level of ambition set by the Member States, which ranges from protecting their neighbourhood to becoming a universal provider of global security -in this respect one notes the Franco-German leadership in defence. German Chancellor Angela Merkel and French President Emmanuel Macron approved a Declaration in June 2018 at the Château de Meseberg that included majority voting in foreign policy, security and defence matters in order to improve the efficiency and effectiveness of decision-making. In addition, it advocated the European Intervention Initiative and new formats such as a European Security Council, as well as strengthening European coordination within the United Nations and the development of a European fighter jet.
\end{abstract}

Based on this context that is outlined in previous lines, in which diverse variables and actors concur and from the perspective offered by the science of international relations, our object of study is articulated that aspires to verify if the changes from a world order based on rules to another truffle of hard power, require a step forward for the European Union with a common strategic culture, analysed from a constructivist approach (Meyer, 2007 y 2004), along with greater leadership and political will. In other words, in the current international context, is ensuring the security and defence of Europe autonomously a necessity for the European Union? Consequently, would NATO support be reduced? At the moment, the Union needs NATO, but it cannot neglect its own capabilities.

In order to articulate our research proposal, we place ourselves in one of the levels of analysis specific to international relations, on the plane of micro-internationality (Calduch, 199I, p. 13), which would be identified with that of the European Union's foreign policy and the challenges it faces. And also in a temporal framework that responds to the line of action that has been developed since the adoption of the Global Strategy in 2016 until the present moment with a Union that is committed to a "geopolitical" Europe.

From an explanatory perspective, supported by consultation of current sources (primary and derived), the analysis is structured as follows: I) The starting point is to examine the complex and uncertain international and European scenario, from which global and regional challenges emerge, with the risk of further conflicts. We explain this context from the instability coming from the East, the Balkans and the Southern Mediterranean, as well as the references to the Trump Administration and the process commonly known as Brexit. 2) To subsequently define what is meant by strategic autonomy in the context of that European Global Strategy and to delimit the variables of analysis. This autonomy has its immediate antecedent in the Saint Malo Agreement (1998). In that agreement, both the United Kingdom and France agreed that the EU should have the capacity for "autonomous action", supported by credible military forces, the means to decide to use them and a preparation to do so.This is with the aim of responding to international crises, and under French-British sponsorship the 1999 Cologne European Council introduced "autonomy of action" with the purpose of acting in international crises included in the Treaties, either when NATO does not do so, as an independent actor, or together with the Atlantic Alliance. 3) The foregoing considerations are complemented and contextualised by the institutionalist approach (Smith, 2004) that provides the study with the different political and institutional instruments that the Union is adopting in the face of the need to promote the generation of civilian and military capabilities, as well as to assess their suitability or otherwise for promoting European strategic autonomy. All of this is aimed at strengthening European unity and giving coherence to external action within the framework of one of its founding pillars, such as the Common Foreign and Security Policy (CFSP) and the European Security and Defence Policy (ESDP), launched at the Cologne Council in 1999 and renamed the Common Security and Defence Policy (CSDP) by the Lisbon Treaty, which called for the adoption of measures to increase EU-NATO cooperation. 4) Finally, fulfilling a practical function, the conclusion is to assess whether or not the Union's evolutionary process in this field is fulfilling its capacities and conditions, and to discern how the next stage should be approached. In this regard, we must assimilate those facts that will impact on the course and pace of the integration process and, therefore, on this desire for strategic autonomy, we refer to the departure of the United Kingdom (Brexit), the economic difficulties of certain European countries of the South in balancing their economic balance and the consequent reaction of the creditor countries of the North, the different perception of the threats and risks of the countries of the North in the face of Russian pressure or those of the South that have to deal with immigration pressure, the expansion of jihadist terrorism or the social and economic crisis caused by the COVID-19 coronavirus pandemic. For its part, the European Council, held in June 2018 , ratified the will of the Member States to continue taking decisive steps to boost European defence, increase strategic autonomy and complement and strengthen NATO activities..

\section{KEYWORDS}

Strategic autonomy; geopolitics; global strategy; European defence; European Union. 


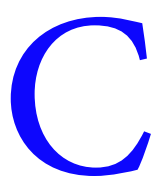

onsideraciones preliminares y contextualización del estudio

Garantizar la seguridad y defensa de Europa de manera autónoma se ha vuelto una necesidad para la Unión Europea (en adelante, UE) tras los acontecimientos geopolíticos que se vienen sucediendo desde 2015. El Brexit, los desafíos en la vecindad Este, Balcanes y Mediterráneo Sur, la llegada de Donald Trump a la Casa Blanca y su hostilidad hacia la UE, la pandemia del coronavirus COVID-19, junto con la asertividad de China en el nuevo orden internacional, obligan a la UE a buscar una mayor unidad política y liderazgo, incluso en un ámbito tan delicado para la soberanía nacional como es la seguridad y defensa. Desde que se puso en marcha, en 2016, la Estrategia Global sobre Política Exterior y de Seguridad de la UE (EUGS, en sus siglas en inglés)' van dando pasos en el ámbito de la seguridad y la defensa, ordenándose hacia el objetivo de la autonomía estratégica. Sin embargo, con una progresión un tanto retraída.

De las necesidades geopolíticas y ante la exigencia de generar más capacidades civiles y militares, se activaron distintos mecanismos: Plan de Acción Europeo de la Defensa (2016), que sentó las bases de una política europea de defensa reforzada²; Fondo Europeo de Defensa, que la Comisión propuso para el marco presupuestario 2021-20273; Cooperación Estructurada Permanente (PESCO, en sus siglas en inglés) puesta en marcha en diciembre de 2017; y, complementariamente, la Iniciativa Europea de Intervención. A los anteriores instrumentos se pueden añadir otros previstos en el Tratado de Lisboa (2009), como son las cláusulas de solidaridad y asistencia mutua, esta última invocada tras el ataque del Daesh en París en noviembre de 20I5, como alianza defensiva para garantizar la seguridad en los países europeos.

Con todo, los desafíos que asolan a la comunidad internacional siguen confrontándose con el deseo de la UE de afianzar su unidad y dar coherencia a la acción exterior en el encuadre de uno de sus pilares fundacionales. Esto es, la Política Exterior y de Seguridad Común (PESC) y la Política Europea de Seguridad y Defensa (PESD), lanzada en el Consejo de Colonia de 1999, que el Tratado de Lisboa renombrará como Política Común de Seguridad y Defensa (PCSD). Sin embargo, no parece claro que Europa tenga la unidad de criterio que requiere aquella política, y la pertenencia a la OTAN de casi todos sus estados miembros hace sentir a muchos dirigentes que es innecesario el esfuerzo que se exige en este ámbito, dado que la arquitectura de seguridad y defensa europea es de por si compleja al estar basada fundamentalmente en dos organizaciones internacionales (OTAN y UE). Asimismo, se debe asimilar aquellos hechos que impactarán en el rumbo y en el ritmo del proceso de integración. Nos referimos a la salida del Reino Unido (Brexit), las dificultades económicas de determinados países europeos del Sur para equilibrar su balanza económica, la distinta percepción de las amenazas y riesgos (de los países del Norte ante la presión rusa o los del Sur que deben lidiar con la presión inmigratoria), la expansión del terrorismo yihadista o el contexto que emerge por la pandemia que la Covid- 19 ha provocado a escala internacional. No sin olvidar que la UE sufrió a partir de 2008 una crisis financiera y económica,

\footnotetext{
Al respecto véase el documento oficial Una visión común, una actuación conjunta: una Europa más fuerte. Estrategia global para la política exterior y de seguridad de la Unión Europea (2016). Recuperado de: https://europa.eu/globalstrategy/sites/globalstrategy/files/eugs_es_version. pdf (26.09.2019). Asimismo, una información detallada de dicha Estrategia Global a los tres años de su implantación se encuentra en https:// eeas.europa.eu/sites/eeas/files/eu_global_strategy_2019.pdf (I0.10.2019).

2 Define las medidas necesarias para una mayor cooperación europea de defensa y apoyar la competitividad de la industria europea y propuso la creación de un Fondo Europeo de Defensa. Véase el apartado 12 de las Conclusiones del Consejo Europeo de I5 de diciembre de 2016.

3 La Comisión presentó el 30 de abril de 2019 una serie de recomendaciones sobre como Europa puede configurar su futuro. Para un conocimiento más exhaustivo, véase Europe in May 2019 Preparing for a more united, stronger and more democratic Union in an increasingly uncertain world. Recuperado de: https://ec.europa.eu/commission/sites/beta-political/files/euco_sibiu_communication_en.pdf (07.I0.20I9).
} 
con profundas ramificaciones sociopolíticas y un auge populista que destacadas repercusiones. Por otra parte, la seguridad energética, los ciberataques, la guerra híbrida o la desinformación también forman parte de los nuevos retos que debe afrontar Europa.

A la vista de lo descrito, la ambición de avanzar hacia una mayor autonomía estratégica es acogida por todos los estados miembros, pero a la vez surgen dudas sobre la viabilidad de la PCSD y la capacidad de la EUGS para impulsarla y que no despierte recelo entre los principales socios internacionales. La autonomía estratégica sitúa su antecedente inmediato en el Acuerdo de Saint Malo (1998), tanto Reino Unido como Francia acordaron que la UE debía tener capacidad de "acción autónoma", apoyada por fuerzas militares creíbles, los medios para usarla y una preparación para hacerlo, con el fin de responder a crisis internacionales. Posteriormente, bajo el patrocinio franco-británico, el Consejo Europeo de Colonia de 1999 introdujo la "autonomía de acción" para poder actuar en las crisis internacionales incluidas en los tratados, bien cuando la OTAN no lo haga como actor independiente, o bien junto con la Alianza ${ }^{4}$.

Para esclarecer estas dudas, partiendo del contexto internacional actual y del concepto de autonomía estratégica que se desprende de los parámetros extraídos de la EUGS, el objetivo del trabajo es abordar el estado de la cuestión desde la perspectiva que nos ofrece la ciencia de las Relaciones Internacionales, para estimar, desde un enfoque constructivista (Meyer, 2004, 2007), si la UE puede lograr la autonomía estratégica en virtud del cambiante entorno geopolítico y valiéndose, en el plano de la microinternacionalidad (Calduch, I99I, p. I3), de los mecanismos políticos e institucionales con los que cuenta la EUGS.Así, podremos responder si la UE está en condiciones de ser suficientemente autónoma asumiendo su propia seguridad y defensa y reduciéndose, consecuentemente, el apoyo OTAN.

\section{La inestabilidad procedente del este, Balcanes y Mediterráneo sur}

Cuando la UE elabora la EUGS, lo hace porque su entorno estratégico cambiante es más complejo, conectado y fragmentado, con un arco de incertidumbre desde el este y el sur con estados fallidos, conflictos en África y tensiones de seguridad en Asia; mientras que el cambio climático y los escasos recursos naturales albergan el riesgo de más conflictos. En el este de Europa, la amenaza de Rusia, tras la anexión ilegal de la península de Crimea y la guerra con Ucrania, la financiación del populismo europeo (Colás, 2017), el uso de la propaganda y la desinformación ${ }^{5}$ (Vilmer et al., 2018), alertó a la UE. Mientras que en el sur y el Mediterráneo, el conflicto civil de Siria y los estados fallidos en los que se convirtieron Irak y Libia dieron lugar a una ola de más de un millón de refugiados hacia Europa, que puso en peligro el sistema de fronteras de Schengen. El terrorismo del Daesh golpeó en varias capitales europeas, borró las fronteras entre seguridad interna y externa y obligó a avanzar en su lucha de manera acelerada.

\section{I. La amenaza rusa en el vecindario del este y los Balcanes}

Los Balcanes y la frontera del este de Europa son prioritarias para la Comisión Europea. El Alto Representante para la Política Exterior, Seguridad y Defensa, Josep Borrell, señala que no seremos creíbles en nuestra ambición de ser un actor geopolítico, "si no somos capaces de resolver los

4 El Acuerdo Berlín plus facilitó esa colaboración en 2003.

5 Un estudio sobre manipulación de la información realizado por el gobierno francés afirma que el $80 \%$ de las autoridades europeas consultadas atribuyen a Rusia interferencias en Europa. 
problemas de nuestra vecindad inmediata" (Parlamento Europeo, 2019a).

A lo largo de su historia, Rusia percibe la aproximación de Bruselas y la OTAN a sus fronteras como intimidatoria para su seguridad (Milosevich-Juaristi, 20I5). Por su parte, el Parlamento Europeo, en la Resolución de 12 de marzo de 2019, no consideraba a Rusia como un "socio estratégico" y le exigía reestablecer la integridad territorial de Ucrania. Dicha resolución apuntaba a la injerencia rusa en procesos electorales, el apoyo del Kremlin a partidos euroescépticos y de extrema derecha o las campañas rusas de desinformación y ciberataques perpetrados por sus servicios de inteligencia para aumentar las tensiones dentro de la Unión.

Los retos de la UE frente a Moscú también provienen de la dependencia energética del gas ruso y busca diversificar el suministro en otros mercados. A pesar de estos esfuerzos, la UE sigue sin poder hablar con una sola voz; un ejemplo de ello es el desacuerdo frente al gasoducto rusoalemán North Stream 2, que no está en consonancia con la política energética de la Unión ni con sus intereses estratégicos al reforzar su dependencia respecto de Moscú.

Pese a la amenaza rusa, el Europarlamento no descarta mantener canales de diálogo con Moscú y subraya que "los retos globales exigen una cooperación selectiva con Rusia", en algunos asuntos. En este entorno, la UE requiere una mayor coherencia en su política exterior y de seguridad con Rusia para defender su integridad territorial y la del espacio postsoviético (este de Europa y Cáucaso), mayor capacidad militar y de despliegue rápido de fuerzas. Para estos fines, la UE cuenta con la OTAN frente a Rusia. Sin embargo, la ambición europea de una autonomía estratégica, en la que el hard power y el soft power van de la mano, es vista por Rusia en el contexto de la Declaración conjunta OTAN-UE en la Cumbre de Varsovia, según la cual la UE está obligada por las directrices políticas y operativas de la Alianza. La Declaración proporciona una respuesta conjunta a desafíos sin precedentes como las amenazas híbridas (Dmitry, 2017), y no deja de ser una respuesta a la anexión ilegal de Crimea, como el despliegue de 4.000 soldados en la frontera norte de la UE.

La política exterior de la UE hacia Rusia produce divisiones internas entre los socios comunitarios. Macron apuesta por reformular la relación de la UE con Rusia como un "primer paso para asegurar la soberanía europea" (Leonard, 2019). Sin embargo, el presidente francés insiste en que Rusia debe respetar los Acuerdos de Minsk en Ucrania y no aboga por levantar las sanciones a Moscú; su acercamiento a Putin para alejarlo de China preocupa en los países Bálticos, Polonia y Rumanía. La influencia de Rusia en los Balcanes también supone una amenaza para la seguridad del continente, tras los intentos del Kremlin de desestabilizar la zona (en Bosnia y Herzegovina) para impedir el acceso de estos países a la OTAN y la ampliación de la UE. Los Balcanes también han despertado el interés de China, que, en 2012, puso en marcha la Iniciativa I6+1, para promover la inversión en infraestructuras ligadas a la Belt Road Iniciative impulsada por Beijing (Grieger, 2018). La Comisión acusa a China de usar financiación barata para encadenar a los países con unas deudas impagables. Las inversiones chinas "suelen descuidar la sostenibilidad socioeconómica y financiera y pueden dar lugar a un alto nivel de endeudamiento y una transmisión del control de activos y recursos estratégicos" (Comisión Europea, 2019). Para evitar la influencia de Rusia, China y de Turquía en los Balcanes, la UE quiere estabilizar la región reanudando el diálogo entre

\footnotetext{
6 Al respecto consúltese el Acuerdo de colaboración y cooperación entre la Unión Europea y Rusia.
} 
Kosovo y Serbia, superando el bloqueo institucional de Bosnia y abriendo las negociaciones para la adhesión de Albania y Macedonia del Norte, a las que la crisis del coronavirus ha dado un impulso tras ver como Serbia pedía ayuda a China.

\section{I.2. Desafíos en el sur y el Mediterráneo}

La vecindad sur es esencial para afrontar la amenaza del terrorismo, los desafíos demográficos, de la migración y del cambio climático.Tras la Primavera Árabe, la EUGS introdujo un nuevo enfoque centrado en la propia seguridad de Europa y en la de su entorno inmediato, sobre todo, tras los ataques terroristas del Daesh y la crisis de los refugiados. La democratización pasa a un segundo plano, debido quizás a esa "realpolitik europea" (Biscop, 2016), que lleva a pactar con regímenes autoritarios para solventar problemas urgentes como en el caso de la coalición frente al Daesh (Benedicto, 2018).

La resiliencia es el nuevo principio rector de las relaciones de la UE con su entorno inmediato y pone fin a la "ingenuidad de la Política Europea de vecindad" (Techau, 2016) y a la capacidad de exportar la democracia. Los estados resilientes son seguros y eso es esencial para la prosperidad y la democracia. Implícitamente, se reconoce que para que suceda un cambio positivo, los estados también necesitan mostrar una voluntad de reforma. De acuerdo con esta visión, "la UE debería contribuir a que los Estados y las sociedades sean más resistentes, como respuesta a una creciente preocupación sobre la proliferación de Estados fallidos, sin gobierno y a los conflictos sectarios en regiones como Oriente Medio o el Norte de África” (Soler i Lecha y Tocci, 2016). La inestabilidad y los conflictos en Oriente Medio y el Norte de África tienen un gran impacto en la UE con la inmigración, la delincuencia transnacional y el terrorismo. La vinculación de las políticas e instrumentos de seguridad externa e interna es una necesidad para contrarrestar estos efectos indirectos $y$, al mismo tiempo, abordar sus causas.

En cuanto a la migración, la detención del flujo masivo de inmigrantes a través del Egeo y el fortalecimiento de la seguridad fronteriza figuraban en primer lugar en la agenda de la UE, a principios de 2016. Sin embargo, debe ir acompañado de medidas para abordar sus causas, como los conflictos, los estados fallidos o la asociación con los países de origen y tránsito, principalmente de África. La Comisión puso marcha, en 2016, un Plan de Inversiones para África y la Vecindad, con el fin de que estos países se comprometiesen a frenar los flujos inmigración y cooperasen en materia de seguridad ${ }^{7}$. Europa prioriza su seguridad y prosperidad, pero para ello necesita estabilizar las regiones vecinas con despliegues y operaciones marítimas en el Mediterráneo, el Índico, o el Golfo de Guinea.Además, será necesaria la protección de fronteras avanzadas como el Sahel y un cinturón de seguridad de Mauritania a Somalia y Sudán.

La presidenta de la Comisión Europea, Ursula Von der Leyen, busca una relación más estrecha con África en un momento de competencia con China. Por su parte, elAlto Representante, Josep Borrell, apuesta por cambiar la relación estrechando la cooperación en cinco puntos: transición verde, digitalización, crecimiento económico, paz y gobernanza e inmigración y movilidad (Comisión Europea, 2020).

\footnotetext{
Véase el Discurso del Estado de la Unión 20/6. Recuperado de: https://ec.europa.eu/commission/priorities/state-union-speeches/stateunion-2016_es (20.01.2020)
} 


\section{Trump y el Brexit, ¿impulsores de la autonomía estratégica?}

En junio de 2016, el Brexit vino a culminar la policrisis europea (Benedicto, 2017), que junto a la llegada de Donald Trump a la Casa Blanca se convirtió en una oportunidad para avanzar en materia de seguridad y defensa.

\section{I. La Administración Trump}

La elección de Trump supuso un cambio en las relaciones transatlánticas al tachar a la OTAN de obsoleta y dar la bienvenida al Brexit.Trump intensificó la presión de Obama para que los estados europeos aumentaran el presupuesto, al señalar que los pagos eran insuficientes, incluso llegando al $2 \%$ del PIB (BBC, 20I7). Un año más tarde, el presidente quería que los estados europeos doblaran el gasto y comprasen a la industria de la defensa de EEUU (Financial Times, 20I8). La confrontación se ha incrementado cuando Washington advirtió que los planes europeos buscan limitar la participación de la industria de defensa americana en los proyectos militares de la UE a través del Reglamento 2018/1092 del Parlamento Europeo y del Consejo, adoptado el I8 de julio de 2018. Este instrumento establece el Programa Europeo de Desarrollo Industrial en materia de Defensa, una norma que permite que participen empresas extracomunitarias, pero exige que la propiedad intelectual del proyecto sea europea y no consiente que terceros países controlen la exportación del armamento fabricado.

Pese a las declaraciones de Trump, la OTAN invitó a Montenegro a ser miembro de la Alianza, y se estableció un puesto de comando atlántico para coordinar una respuesta rápida de la Alianza en caso de, por ejemplo, una guerra en Europa entre Rusia y los aliados de la OTAN. Los países de la Alianza también elaboraron un acuerdo de movilidad para permitir que sus fuerzas avancen rápidamente a través del territorio de la alianza en toda Europa con el fin de poder responder a una hipotética agresión rusa. Pero, como explica Arteaga (2018), quizás no sea más que una cortina de humo para ocultar el debilitamiento real del lazo transatlántico, cuyos valores a ambos lados ya no parecen ser los mismos que antaño. Existe una especie de distanciamiento transatlántico que va más allá del asunto del gasto militar y tiene más que ver con el giro de EEUU hacia Asia o con el rechazo de la Administración Trump al acuerdo nuclear con Irán o a su salida del acuerdo del cambio climático o del tratado de control de armas atómicas con Rusia. La irrupción de Trump parece jugar como federador externo de la UE en el campo de la seguridad y defensa. Como señaló la canciller alemana,Angela Merkel, tras las cumbres del G-7 y de la OTAN, la UE no puede depender de un líder cada vez menos fiable (en referencia a Trump).

\subsection{La seguridad y defensa europea tras la salida de Reino Unido}

El Brexit tiene consecuencias complejas para la defensa Europa. Reino Unido, pese a los obstáculos que siempre ha puesto a los avances de la PCSD, era la mayor potencia militar de la Unión y su salida puede perjudicar la relación con la OTAN, a la seguridad y a las capacidades de defensa europeas. Sin embargo, el divorcio de Londres tiene otra lectura: su papel de federador interno al impulsar con su salida una defensa europea más autónoma (Benedicto, 2019). Tras el anuncio del Brexit, se puso en marcha la PESCO y un Cuartel General para operaciones no ejecutivas, algo a lo que Londres siempre se oponía.

En efecto, la salida de Londres perjudica la defensa de la UE al contar con menos recursos 
para el Fondo Europeo de Defensa y al "perder el $20 \%$ de sus capacidades militares y el $40 \%$ de sus capacidades industriales de defensa, y por lo tanto su influencia y credibilidad como actor de seguridad" (Round, Giegerich y Mölling, 2018). Reino Unido reconoce la necesidad de seguir vinculado a Europa en asuntos de defensa, pues "sería una asociación para hacer frente a las amenazas comunes, y promover los valores que compartimos en todo el mundo y mantener una Europa segura y próspera" (Gobierno de Reino Unido, 2017). Tanto Reino Unido como la UE podrían trabajar lo más cerca posible "para salvaguardar una industria de defensa sostenible, innovadora y competitiva, que forma parte de los intereses estratégicos de todos los socios políticos en Europa” (Schüt y Mölling, 2018). La PESCO y la definición de los criterios de participación de terceros estados en sus proyectos, como la Iniciativa para la Intervención Europea, podrían ser la solución para incluir a Londres de manera explícita la defensa europea.

\section{Concepto de autonomía estratégica en el marco de la Estrategia Global europea}

No hay una definición común y uniforme para el concepto de autonomía estratégica dentro de la Unión Europea, ni tan siquiera un documento oficial que la especifique y la regule como tal. Sin duda, un concepto tan generalizador, como el de autonomía, puede conducir a análisis diversos según el marco teórico de referencia (Tokatlian y Carvajal, 1995). Desde un punto de vista teórico, para Grevi (2019) no es solo un asunto de política exterior o de seguridad y defensa, sino que es un "requisito crítico para sostener e impulsar la integración europea. Construir una Europa más autónoma no es solo protegerla mejor sino también proyectar una agenda positiva en el escenario mundial"'. Siguiendo a Grevi (2019), la autonomía estratégica engloba tres dimensiones principales: económico, tecnológico y de seguridad y defensa. El presente estudio se articula en torno al tercer dominio.

Dependiendo del autor consultado, la autonomía estratégica es la capacidad de defender su propio territorio o ser capaz de defender su vecindad e incluso jugar un papel como actor global (Howorth, 2019), o puede referirse a una mayor dependencia de la industria europea de defensa en términos de suministro de capacidades militares para los estados miembros (Lawerence, Praks y Järvenpää, 2017), o a la habilidad de llevar a cabo operaciones militares exigentes en el continente europeo (Varga, 2017). Mientras que, si atendemos a las estrategias de defensa de los distintos países, el concepto de autonomía estratégica varía mucho de uno a otro.Al respecto, los estados de la UE no están de acuerdo con el nivel geográfico y funcional de ambición que deberían adoptar para alcanzar la autonomía estratégica. Para Francia es la capacidad de decidir y actuar libremente en un mundo interdependiente (República Francesa, 2017), mientras que Polonia la percibe como el intento de algunos Estados de poner fin a la dependencia de EEUU (Drent, 20I8), y por eso quiere que se complemente con la OTAN. Esa concepción propia, a nivel de estado, y tan variada es lo que ha llevado a que la EUGS no especifique su significado concreto

\footnotetext{
8 En efecto, siguiendo a los autores citados en el texto, "el desacuerdo sobre el significado de la autonomía se encuentra en ese nivel de las concepciones: ¿Cuáles son las variables que definen la autonomía? ¿Qué cuantía de ciertas variables y requisitos se necesita para que un país pueda ser considerado autónomo? ¿Es posible o viable ser autónomo? ¿Es la autonomía un valor deseable per se? ¿Puede hablarse indistintamente de un mismo tipo de autonomía en y para los países centrales y en y para los países periféricos? A éstos, podríamos sumar otros tantos y más interrogantes sobre las concepciones de autonomía; lo que con seguridad nos conduce a observar y detectar una generosa gama de respuestas y matices [...]" (Tokatlian y Carvajal, 1995, p. 8).

9 El estudio de la autonomía en política exterior puede abordarse, de manera introductoria, desde la óptica de algunas teorías de las relaciones internacionales. Un tratamiento resumido relativo a la autonomía de los países periféricos desde la perspectiva de los diversos paradigmas que conviven en la ciencia de las Relaciones Internacionales se encuentra en Potter, D. (1992). The Autonomy of Third World States within the Global Economy. En McGrew, A.G.y Lewis, P.G. (Eds.) (1992). Global Politics. Cambridge: Polity Press.
} 
y lo deje vago y ambiguo ${ }^{10}$.

Asimismo, advertir que cuando hablamos de autonomía estratégica no estamos pensando en un ejército europeo común debido a las diferentes culturas estratégicas y percepciones de los Estados miembros", pues hay países más intervencionistas como Francia y los hay menos proclives al uso de la fuerza como Alemania (Franke y Varma, 2019). A pesar de la indefinición que se evidencia, en la Estrategia se menciona por primera vez de manera explícita la autonomía estratégica como un objetivo de la UE. En el prólogo de la nueva estrategia adoptada en 2016 se expone la ambición de conseguir "una autonomía estratégica para la Unión Europea" y de contar "con la fuerza para contribuir a la paz y la seguridad en la región y en todo el mundo". En efecto, existe la intención de adquirir una capacidad propia en materia de defensa y contribuir a la seguridad colectiva. Se trata de "un paso importante para el pensamiento estratégico europeo" e incluso así ha sido visto desde Estados Unidos cuando critican de manera unánime que puede socavar a la OTAN (Biscop, 2019).

El término "autonomía" figura mencionado siete veces en la Estrategia, al referirse a la toma de decisiones y a la "autonomía de acción" (Franke y Varma, 2019). En dicho documento se subraya que es importante fomentar "un apropiado nivel de ambición y de autonomía estratégica para impulsar la paz y la seguridad en Europa y más allá de sus fronteras". Asimismo, se deduce del plan de implementación de la Estrategia Global esta voluntad de autonomía, que no es incompatible con la Alianza, sino que, desde el punto de vista europeo, la refuerza, cuando resalta que es necesaria dicha autonomía para "promover los intereses comunes de nuestros ciudadanos, así como nuestros principios y valores"; o al enunciarse que se apuesta por "una industria de defensa europea innovadora y competitiva" como algo esencial para ser autónomos. En el balance que hizo la Comisión tres años después del lanzamiento de la EUGS, se destaca que "el trabajo realizado ha establecido unos cimientos sólidos para la Unión Europea de la Seguridad y la Defensa y para su autonomía estratégica”.

Al respecto, estimamos que la autonomía estratégica es la capacidad de actuación autónoma (civil y militar) de la Unión en cualquier conflicto, con sus propias herramientas de seguridad y de defensa, con socios internacionales y regionales, sin necesidad de que la OTAN intervenga en todo caso. Este planteamiento se traduce en la exigencia de que Europa pueda defenderse de agresiones de terceros sin tener que depender exclusivamente de la Alianza Atlántica o de un aliado como es Estados Unidos con quien comparte intereses y rivalidad. El desarrollo de las capacidades de los estados miembros a través de la PCSD y el uso de instrumentos de la UE también ayudará a fortalecer las capacidades potencialmente disponibles para las Naciones Unidas y la OTAN.

Las consideraciones anteriores nos conectan con otro concepto, no menos importante,

\footnotetext{
10 Cualquier actor con autonomía estratégica debe tener tres dimensiones: autonomía política, operativa e industrial. La autonomía operativa se define como la capacidad para planificar y realizar independientemente operaciones civiles y/o militares. La autonomía industrial significa la habilidad de desarrollar las capacidades requeridas para lograr la autonomía operativa. Estos dos elementos deben complementarse con la autonomía política, que es la capacidad de definir objetivos de política exterior y de seguridad y decidir sobre las herramientas que se utilizarán en su búsqueda (Kempin y Kunz, 20I7, p. 10). Por su parte, otros académicos incluyen la autonomía nuclear que, de momento es un tabú para la UE, pese a que EEUU podría en un momento dado dejar de proporcionar el paraguas atómico y Europa necesitar su propio poder nuclear (Drent, 2018).

" Para Martinsen (2003) "son las ideas, expectativas y patrones de comportamiento que se comparten entre los actores involucrados en los procesos que rodean las políticas europeas de seguridad y defensa".
} 
como es el de cultura estratégica. Siguiendo a Meyer (2004), la cultura estratégica es una variable que interviene en el análisis de la política exterior para comprender el comportamiento de los Estados y organizaciones internacionales en asuntos de seguridad y defensa. Su relevancia puede entenderse mejor desde una perspectiva constructivista dentro de las Relaciones Internacionales, un enfoque que se desarrolla en torno a preguntar si está surgiendo una cultura estratégica europea común o si de hecho ya existe de alguna forma y qué efectos tiene. Para que la política exterior de seguridad y defensa vaya más allá, "requerirá de una base de intereses, valores y prioridades compartidos, percepciones de amenaza y medios legítimos para el uso de la fuerza militar, así como un acuerdo sobre el papel de Europa en el mundo" (Meyer, 2007). Si el desacuerdo - la divergencia en estos temas persiste, algunos académicos como Krotz y Maher (200l) creen, "que la cooperación en estas áreas políticas es poco probable que se desarrolle y consolide".

En definitiva, observamos que, en el entorno de inestabilidad e incertidumbre en que nos encontramos y dada la confluencia de políticas internas y externas, la Unión debe actuar de forma coordinada y coherente para garantizar sus principios, sus valores e intereses en tiempos de cambio. Es por ello que adquiere lógica el enfoque integrado adoptado por la EUGS, y que enfatiza las acciones de prevención, gestión y resolución de conflictos, a diferencia de la anterior Estrategia de Seguridad centrada solo en la prevención de conflictos. De todo lo anterior, subyace que la autonomía pueda ser viable y benéfica. Pero esta viabilidad se alcanza con la conjunción de dos factores estructurales fundamentales (Simonoff y Lorenzini, 2019): la posesión de recursos suficientes mínimos (en la expresión de Jaguaribe), y la existencia de "elites funcionales, o sea, decididas a emprender el camino de la autonomización” (Puig, 1980, p. 154). Sin olvidar, la "permisibilidad internacional", que:

"Se refiere fundamentalmente a la medida en que, dada la situación geopolítica de un país y sus relaciones internacionales, este país disponga de condiciones para neutralizar el riesgo proveniente de terceros países, dotados de suficiente capacidad para ejercer sobre él formas eficaces de coacción -como el desarrollo de una apropiada capacidad económico-militar, o también externas, como el establecimiento de convenientes alianzas defensivas" (Jaguaribe, 1979, p. 97).

\section{Nuevas herramientas institucionales y políticas para la seguridad y defensa europea} Tras la aprobación de la EUGS, la UE desarrolló una serie de nuevas herramientas e instituciones con el fin de intensificar la cooperación en materia de seguridad y defensa europea. Para su estudio se aplica el enfoque institucionalista de Smith (2004), que explica como la política exterior europea y la cooperación en seguridad surgieron a través de "la institucionalización de hábitos y patrones de cooperación, consulta y construcción de consenso". Esta institucionalización ha afectado no solo los estados miembros, que se consultan unos a otros antes de actuar unilateralmente, sino también al sistema institucional europeo y a los dirigentes y funcionarios que trabajan desde Bruselas.

En estos cuatro últimos años, los estados miembros incrementaron la cooperación en defensa mediante el desarrollo de nuevos instrumentos tras la cumbre de Bratislava en 2016 y después del Consejo Europeo (véase el Plan de Aplicación en el ámbito de la seguridad y la defensa); y también en la Comisión Europea con la creación del Fondo Europeo para la 
Defensa y de una Dirección General del Espacio e Industria de Defensa en el ejecutivo Von der Leyen, que introducen cambios innovadores en un área política fragmentada y compleja, donde el intergubernamentalismo sigue siendo dominante y la gobernanza supranacional ha sido tradicionalmente limitada.

\section{I. El Plan de Aplicación en el ámbito de la seguridad y la defensa}

El Plan se centra en tres prioridades: dar respuesta a los conflictos y las crisis exteriores, aumentar las capacidades de los socios y proteger a la UE y a sus ciudadanos. Para alcanzar esos objetivos, se puso primero en marcha la Revisión Anual Coordinada de la Defensa (CARD, en sus siglas en inglés) para armonizar la planificación de la defensa con el intercambio voluntario de información sobre los planes de defensa nacional y las contribuciones al Plan de Desarrollo de Capacidades (CDP) de la Agencia Europea de Armamento. Todo ello permitirá tener una visión general más clara del gasto a escala de la UE, con el fin de resaltar las oportunidades de colaboración, evitar duplicidades y rellenar vacíos en asuntos como los gastos de defensa e inversión nacional, así como los esfuerzos de investigación de defensa (Guinea, 2018). En cuanto a los instrumentos de respuesta rápida se reforzaron los battle groups, creados en 2005 y nunca utilizados, para responder rápidamente a crisis y conflictos, asumiendo su despliegue como un coste financiero común de la UE.

La UE desplegó cerca de cuatro mil efectivos en 2019, en seis misiones militares y diez misiones civiles. Operaciones militares como Atlanta para luchar contra la piratería en el Cuerno de África y mantener rutas comerciales marinas abiertas y seguras ha sido un éxito. Otras, como la Operación Sophia ${ }^{12}$, que se desplegó en 2015 para evitar el tráfico de migrantes en el Mediterráneo, quedó neutralizada en marzo de 2019 por el bloqueo del gobierno de Italia, tras el cierre del sistemático del acceso de los buques a puertos italianos. La salida del vicepresidente Matteo Salvini del ejecutivo italiano reanudó esta misión, pero reconvertida en hacer efectivo el embargo de armas a Libia.

Por su parte, el Plan de Aplicación reforzó la capacidad de la UE para el despliegue de misiones civiles $^{13}$ de gestión de crisis; con el fin de desplegar hasta doscientos efectivos en cualquier zona de operaciones en un plazo de treinta días a partir de una decisión del Consejo ${ }^{14}$. Por último, también se creó una Capacidad Militar de Planificación y Ejecución, que a finales de 2020 se quiere que asuma una operación ejecutiva limitada al tamaño de un grupo de combate europeo (unos dos mil quinientos efectivos); y un Cuartel Europeo con mando en las misiones militares no ejecutivas ${ }^{15}$.

\footnotetext{
12 Véase: Consejo Europeo (20.03.2019). Operación EUNAVFOR MED SOPHIA: se prorroga el mandato hasta el 30 de septiembre de 2019. Recuperado de: https://www.consilium.europa.eu/es/press/press-releases/2019/03/29/eunavfor-med-operation-sophia-mandate-extendeduntil-30-september-2019/ (07.06.2020).

13 Su objetivo es reforzar la policía, el Estado de Derecho y la administración civil en entornos frágiles y de conflicto.

14 Véase: Consejo Europeo (19.1 I.2018). Conclusiones del Consejo y de los Representantes de los Gobiernos de los Estados miembros, reunidos en el seno del Consejo, relativas a la adopción de un Pacto sobre la Vertiente Civil de la PCSD. Recuperado de: http://register.consilium.europa.eu/content/out? lang $=E S \&$ tyP $=S E T \& i=A D V \& R O W S P P=25 \& R E S U L T S E T=\mid \& N R R O W S=500 \& D O C \_L A N C D=E N \& O R D E R B Y=D O C \_D A T E+D E S C \& D O C$

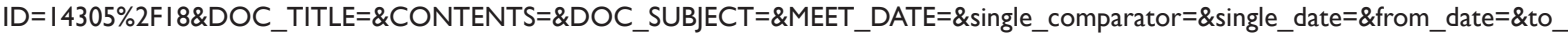
date $=(07.06 .2020)$.

15 Como las de entrenamiento en la República Centroafricana, Malí y Somalia, que cuenta con un equipo de veinticinco personas.
} 


\subsection{Plan de Acción Europeo de Defensa}

La UE contaba, en 2015, con 203.000 millones de euros destinados a la defensa, lo que le situaba en segundo lugar a escala mundial en gasto militar, después de los Estados Unidos. Sin embargo, esos fondos con frecuencia se utilizaban de manera poco eficiente debido a la fragmentación del mercado europeo de defensa, la duplicación de capacidades militares, la insuficiente colaboración industrial y la ausencia de interoperabilidad. Para afrontar estos problemas, la Comisión presentó el Plan de Acción Europeo de Defensa para mejorar la ecuación coste-eficacia del gasto en defensa, aumentar la cooperación y crear una base industrial más sólida. El Plan se articuló entorno a tres pilares: la creación de un Fondo Europeo de Defensa (FED), el fomento de las inversiones en la industria y el refuerzo del mercado único de la defensa. El FED supone la incorporación de la Comisión Europea a la Defensa para incentivar a los Estados miembros a gastar más en investigación y desarrollo de capacidad de defensa, colocar a la UE entre los principales inversores en investigación y tecnología de defensa en Europa y alentar un mayor gasto por parte de los Estados miembros (Besch, 2019). En este sentido, Csernatoni (2019) estima que, "simboliza un desarrollo sin precedentes de la gobernanza de la UE a nivel supranacional: hace realidad la cooperación industrial de defensa bajo el presupuesto de la UE”.

\subsection{La Cooperación UE-OTAN}

En el entorno estratégico actual la cooperación entre la UE y la Alianza es fundamental pues la seguridad de las dos organizaciones está interconectada. La cooperación UE-OTAN se hace con pleno respeto de la autonomía decisoria y de los procedimientos de ambas y sin perjuicio del carácter específico de la política de seguridad y defensa de los Estados miembros. Veintidós estados de la Alianza también son miembros de la UE, y juntos pueden movilizar una amplia gama de herramientas y hacer el uso más eficiente de los recursos para abordar esos desafíos y mejorar la seguridad de sus ciudadanos. Los veintisiete han dibujado las líneas de la hoja de ruta hacia la autonomía estratégica, pero no parece viable a corto plazo. De ahí que la EUGS hable de complementariedad entre la UE y la OTAN. Todo ello contribuye, sin duda, al reparto transatlántico de la carga.

La cooperación entre ambas organizaciones desde 2003, tras la firma de los Acuerdos de Berlín Plus, permiten a la UE hacer uso de los medios y de las capacidades de la OTAN para las operaciones de gestión de crisis dirigidas desde la Unión. Esa coordinación ha sido mucho más intensa tras la anexión de Crimea a Rusia y, en los últimos tres años, por los desafíos a la seguridad en el sur de Europa. Durante la Cumbre de Varsovia de 2016, se adoptaron una serie de medidas de disuasión y defensa, para el restablecimiento de la seguridad en Europa oriental ${ }^{16}$.

La UE y la OTAN firmaron, en julio de 20I8, una nueva declaración conjunta, que constituye una visión compartida sobre el modo en que actuarán frente a las amenazas para la seguridad común. Aun así, Europa debería preparar un plan B para garantizar, como dijo Merkel, que puede asumir su destino, independientemente de quién esté en el poder en la Casa Blanca. La UE debería ser un actor con autonomía estratégica, quizás no solo para operaciones expedicionarias sino también para la defensa territorial, lo que conduciría a lo que Biscop (2018, pp. 85-93) denomina,

\footnotetext{
16 Véase: Comisión Europea (08.07.16). Joint Declaration by the President of the European Council, the President of the European Commission, and the Secretary General of the North Atlantic Treaty Organization. Recuperado de: https://ec.europa.eu/commission/presscorner/detail/en/ STATEMENT_16_2459
} 
reemplazar a la OTAN con una nueva alianza EEUU-UE. Pero, ¿conviene sustituir a la OTAN? Al respecto, en su discurso de investidura ante el Parlamento Europeo, la presidenta Von der Leyen subrayó que "la piedra angular de nuestra defensa colectiva siempre será la OTAN.Aunque seguiremos siendo transatlánticos, tenemos que volvernos más europeos. Por eso creamos la Unión Europea de Defensa" (Comisión Europea, 2019, p. 9). Por su parte, el Alto Representante de la UE, Josep Borrell, en su audiencia ante el Europarlamento, también apostó por reforzar la OTAN, pero para que la UE sea "geopolíticamente más relevante será necesario aumentar nuestra capacidad militar para actuar" (Parlamento Europeo, 2019).

\subsection{La Cooperación Estructurada Permanente}

El Tratado de Lisboa dispone que un grupo de al menos nueve estados miembros que deben cumplir criterios más elevados de capacidades militares ${ }^{17}$, reforzando su cooperación en defensa ${ }^{18}$. Desde 2018, los veinticinco estados participantes han aprobado cuarenta y siete proyectos que abarcan desde un Comando Médico Europeo a equipos de respuesta rápida cibernética.

La PESCO se relaciona, por un lado, con la CARD, que hace una evaluación periódica de las contribuciones nacionales a la defensa de cada estado; y, por otro lado, con el Fondo Europeo de Defensa, que ofrece incentivos financieros para que los estados fomenten la cooperación en investigación. La diferencia entre PESCO y otras formas de cooperación es la naturaleza vinculante de los compromisos realizados por el estado miembro participante, que participa de modo voluntario, manteniendo su toma de decisiones y el carácter específico de su política de seguridad y defensa.

Por otra parte, el Consejo reconoció que un tercer estado podría aportar valor añadido a los proyectos de la PESCO y de la PCSD, sin perjuicio del respeto al principio de autonomía decisoria de la UE y de sus Estados miembros. Conforme a esta excepción, se podría dar entrada al Reino Unido.

\subsection{La Iniciativa Europea de Intervención}

El presidente francés, Emmanuel Macron, propuso la Iniciativa Europea de Intervención (IEI), para fomentar una cultura estratégica europea que contribuya a la PESCO ( $y$ a la Alianza) y, en particular, reforzar la capacidad de los europeos para actuar juntos. Su misión es dar una respuesta rápida y eficiente a las crisis que puedan amenazar la seguridad europea, tanto fuera como dentro del territorio europeo. Además, es una estructura intergubernamental creada fuera de la UE y de la OTAN. Los Estados firmantes podrían formar coaliciones e intervenir de manera rápida y efectiva cuando surja una crisis bajo el paraguas de la UE, la OTAN, ONU o de coaliciones ad hoc.

Diez países europeos, al margen del Consejo Europeo, han demostrado su voluntad política y sus capacidades militares para asumir su compromiso en operaciones militares ${ }^{19}$. Para algunos, la IEI pretende complementar tanto a la OTAN como a la UE. Macron la puso en marcha

\footnotetext{
17 Definidos en el Protocolo $\left(n^{\circ} 10\right)$ sobre la cooperación estructurada permanente establecida por el artículo 42 del Tratado de la Unión Europea.

18 Véase: Consejo de la Unión Europea (17.06.2019). Council Conclusions on Security and Defence in the context of the EU Global Strategy - Council Conclusions. Recuperado de: https://www.consilium.europa.eu/media/39786/st I 0048-en I 9.pdf (07.06.2020)

19 Bélgica, Dinamarca, Estonia, Francia, Alemania, los Países Bajos, Portugal, España, Reino Unido y Finlandia.
} 
"molesto con la relativa falta de ambición de PESCO, y como un movimiento para promover una arquitectura de defensa europea que se adapte a los intereses de Francia” (Moya, 2019). Será importante advertir que la IEI no forma parte de la PESCO para permitir la participación sin restricciones de países con capacidad militar y políticamente dispuestos, como Dinamarca (cuya exclusión voluntaria de la PCSD obstaculiza cualquier participación en PESCO), o como Reino Unido, o tal vez a otros países en el futuro.

\section{Liderazgo francoalemán en defensa}

Sin el peso del eje francoalemán y tras la salida del Reino Unido, es difícil que salga adelante cualquier política europea de calado. Pese a que Alemania tiene una visión de la defensa más inclusiva y es partidaria de la PESCO, y Francia prefiere el modelo de integración de defensa "de varias velocidades" de la Iniciativa Europea de Intervención, tanto Merkel como Macron han ido impulsando la Defensa Europea, para reforzar la capacidad de acción autónoma de Europa.Ambos aprobaron, en junio de 2018, la Declaración de Meseberg que recogía el voto por mayoría en materia de política exterior, seguridad y defensa, allí donde los tratados lo hacen posible, para mejorar la eficacia y efectividad en la toma de decisiones. la Iniciativa Europea de Intervención, nuevos formatos como un Consejo Europeo de Seguridad, reforzar la coordinación europea en el seno de las Naciones Unidas y un caza de combate europeo. Merkel en su discurso ante el Parlamento Europeo, en noviembre de 2018, desarrolló esas ideas, como la de un Consejo de Seguridad Europeo con miembros rotativos de los estados miembros, en el que se puedan preparar decisiones importantes con mayor rapidez; y abogó por una fuerza de intervención europea con la que Europa pueda actuar localmente e, incluso, por trabajar en la creación de "un verdadero ejército europeo" pero complementario a la OTAN. Macron también apoyó la idea del ejército, según se extrae del testimonio recogido en el medio Europe $I$, el 6 de noviembre de 20 I8; y fue más allá en unas declaraciones formuladas en The Economist, el 7 de noviembre de 2019, al hablar de "muerte cerebral" de la OTAN tras la retirada de EEUU del norte de Siria. Macron advirtió que Europa “desaparecería geopolíticamente" a menos que comenzara a actuar como un poder estratégico.

Con todo, el pacto francoalemán llevó a Von der Leyen a la presidencia de la Comisión, que apuesta por una Europa "geopolítica" que trae connotaciones de un enfoque de política exterior centrado en la distribución de hard power: poder militar y económico. En línea con este planteamiento, en su audiencia ante el Parlamento (2019a), el Alto Representante Josep Borrell señaló que la UE debe "aprender a usar el lenguaje del poder" y necesita "cultura estratégica".

\section{Conclusiones}

Pese a que desde la aprobación delTratado de Lisboa se han generado instrumentos para que la UE despliegue su autonomía estratégica con estructuras de toma de decisiones autónomas (enfoque institucionalista), todavía se está trabajando en el desarrollo de las capacidades civiles y militares necesarias para las operaciones y en una industria de defensa europea que permita producir esas capacidades. Contando con el amparo de la EUGS, estimamos que aún queda camino por recorrer y voluntad política común para que los intereses de los Estados miembros converjan en una cultura estratégica común. Desde el enfoque constructivista podemos ver que, pese a que las culturas estratégicas en la Unión son diferentes entre los países miembro, el 75\% de los europeos apoya una política común de defensa y seguridad, y una mayoría (55\%) está a favor de la creación 
de un ejército de la UE (Eurobarómetro Especial 46I, 2017), mientras que en el Eurobarómetro 89.2 (2018), el $68 \%$ de los europeos dijo que les gustaría que la UE hiciera más en defensa.

En un entorno geopolítico que se torna complejo y en el que es necesario tener en cuenta políticas de poder, la nueva Comisión apuesta por reforzar el papel internacional del euro y aumentar la capacidad militar para actuar. Sin duda, las contrariedades a las que debe hacer frente la UE requieren mayor unión política, liderazgo, una visión estratégica compartida, mayor coherencia entre las iniciativas que se han puesto en marcha (PESCO, Iniciativa Defensa Estratégica, FED y CARD) e identificar el nivel militar de ambición que se persigue.

La Unión progresa tímidamente para demostrar a la comunidad internacional que es capaz de hacer frente con autonomía y solvencia a las amenazas que cercenan la seguridad y la paz mundial, de ser competente para ayudar a sus socios y de proteger su territorio y a sus ciudadanos, de forma complementaria con la OTAN. Sin embargo, frente a esa sutil progresión, la actualidad nos muestra episodios de involución que restan credibilidad al discurso de los máximos dirigentes y cercenan la confianza para demostrar esa capacidad de autonomía. En este caso, no basta con parecer sino también con ser.

La retirada gradual de Estados Unidos de Europa y de Oriente Medio y su apuesta por el proteccionismo coincide con una mayor asunción de responsabilidades en Bruselas. Pero a corto plazo, es evidente que la UE necesita a Washington para su defensa territorial, pues todavía se deja en manos de la OTAN la defensa territorial y las operaciones que requieran grandes despliegues sobre el terreno. No parece creíble una Europa sin la Alianza de aquí a quince años o más. Por ello, lo óptimo es mantener los lazos con la OTAN mientras se desarrolla la autonomía estratégica. Además, Bruselas desea el anclaje del Reino Unido en la seguridad y defensa europea a través de mecanismos como la PESCO o la IIE e incluso con en un futuro Consejo de Seguridad Europeo. Por su parte, en el vecindario sur, parece que Europa está dispuesta a asumir la autonomía, jugando un papel destacado a medio plazo. Reiteramos, no basta con parecer sino también con ser. Mientras que en el vecindario del este (Rusia), la OTAN sigue siendo la fuerza de disuasión a la que la UE está subordinada.

Dibujar un horizonte autónomo es tarea harto complicada frente al papel preponderante de EEUU, la amenaza terrorista, los desplazamientos migratorios, los efectos del Brexit o el impacto social y económico de la crisis del coronavirus en el presupuesto europeo para defensa, por citar algunos. Pero no es un cometido imposible, siempre y cuando aflore una verdadera voluntad política común entre los estados miembros de ir más allá de una leve autonomía y, aún más, que los otros actores protagonistas de la escena internacional también permitan este desarrollo estratégico europeo.

Con todo, es vital para la supervivencia de la UE como actor global que gane su independencia en actuación exterior como líder mundial de prevención de conflictos. Es por ello que Europa debe combinar sus capacidades civiles y militares para la agenda estratégica 20192024, definir nuevos objetivos, ambiciosos, realistas y específicos para este nuevo ciclo político, y la cooperación a todos los niveles es la única manera de responder a las expectativas de los ciudadanos europeos. Nadie cuestiona la necesidad de una UE con mecanismos civiles y militares 
para mantener y proyectar estabilidad en la comunidad internacional, ni su cualidad de ser creíble en la escena económica, pero si se cuestiona su destreza para ser un actor con voz sólida y verosímil en la actual reconfiguración del orden mundial. Pese a los avances logrados, para mejorar su capacidad y actuar como proveedor de seguridad, habrá que estar pendientes de la apuesta por una Europa geopolítica de la nueva Comisión.

\section{Referencias}

Arteaga, F. (I2.09.20 I8). No es el 2\%, json las relaciones transatlánticas, estúpidos! Blog Real Instituto Elcano. Recuperado de: https://blog.realinstitutoelcano.org/no-es-el-2-son-las-relaciones-transatlanticas-estupidos/ (I2.1 I.2019).

Benedicto Solsona, M. A. (2019). La contribución federal de la Política de Seguridad y Defensa. En Bengoetxea, J. (Coord.) Europa de las regiones y el futuro federal de Europa. Balance y perspectiva de la gobernanza multinivel de la Unión Europea (pp. 157-172). Madrid: Dykinson.

Benedicto Solsona, M.A. (2018). Seguridad y Defensa de la UE en el Mediterráneo Sur. En Priego,A.y Bay Rasmussen, S. (Coords.). La proyección exterior de la UE hacia el Mediterráneo Sur tras las Primaveras Árabes (pp. 99-124). Navarra: Aranzadi.

Benedicto Solsona, M. A. (2017). Pasos de gigante en la defensa europea. Política Exterior, 3 I (I75), I06-I I5.

Besch, S. (2019). The European Commission in EU Defense Industrial Policy. Carnegie Europe. Recuperado de: https://carnegieeurope.eu/2019// 0/22/european-commission-in-eu-defense-industrial-policy-pub-80 I02 (I2.04.2020).

Biscop, S. (2019). Fighting for Europe. European Strategic Autonomy and the use of force. InEgmont Papers, 103. Recuperado de: http://www.egmontinstitute.be/content/uploads/20I9/0I/EPI03.pdf?type=pdf (07.06.2020).

Biscop, S. (20/8). EU-NATO relations: a long-term perspective. Nação e Defesa, I50, 85-93.

Biscop, S. (2016). The EU Global Strategy: Realpolitik with European Characteristics Security. Policy Brief, 75. Recuperado de: http://www.egmontinstitute.be/content/uploads/20 I6/06/SPB75.pdf?type=pdf (07.06.2020).

Calduch Cervera, R. (1991). Concepto y método de las relaciones internacionales. En Relaciones Internacionales (pp. I-20). Madrid: Ediciones Ciencias Sociales. Recuperado de: https://www.ucm.es/data/cont/media/www/pag55I59/lib I cap I.pdf (I5.04.2020).

Colás, X. (25.03.2017). Vladimir Putin y Marine Le Pen, el eje antieuropeo. Diario El Mundo. Recuperado de: https:// www.elmundo.es/internacional/20I7/03/25/58d57d I lca474I08I28b4600.html (I0.04.2020).

Comisión Europea (16.07.2019). Opening Statement in the European Parliament Plenary Session by Ursula von der Leyen, Candidate for President of the European Commission. Recuperado de: https://ec.europa.eu/commission/ presscorner/detail/en/SPEECH_19_4230

Comisión Europea (2019). European Commission and HR/VP contribution to the European Council. EU-China- A strategic Outlook. Recuperado de: https://ec.europa.eu/commission/sites/beta-political/files/communication-eu-chinaa-strategic-outlook.pdf (07.06.2020).

Comisión Europea (09.03.2020). Comunicación conjunta al Parlamento Europeo y al Consejo. Hacia una estrategia global con África. Recuperado de: https://op.europa.eu/es/publication-detail/-/publication/558I7dfb-6 l eb-I l ea-b735 -0 laa75ed7lal (07.06.2020).

Cornish, P. y Edwards, G. (2005). The Strategic Culture of the European Union: A Progress Report. International Affairs, $8 \mathrm{I}(4), 80 \mathrm{I}-820$.

Csernatoni, R. (2019). The Democratic Challenge of EU Defense Policy. Carnegie Europe. Recuperado de: https://carnegieeurope.eu/strategiceurope/80384 (I2.04.2020).

De Miguel, B. (I2.05.20I9). EEUU amenaza a Europa con represalias si impulsa en solitario sus proyectos de Defensa. El País. Recuperado de: https://elpais.com/internacional/2019/05/I2/actualidad/I5576625I7_97498I.html $(10.10 .2019)$.

Dmitry, D. (2017). EU-Russia Relations in the Post-Soviet Space. En Biscop, S. The EU global strategy: implications for Russia (pp. 15-25). Moscú: Egmont Royal Institute for International Relations and Institute of Europe.

Drent, M. (08.08.2018). European strategic autonomy: going it alone. Clingendael. Recuperado de: https://www. clingendael.org/publication/european-strategic-autonomy-going-it-alone (07.10.2019).

Emmnuel Macron warns Europe: NATO is becoming brain-dead (07.II.2019). The Economist. Recuperado de: https:// www.economist.com/europe/2019/I I/07/emmanuel-macron-warns-europe-nato-is-becoming-brain-dead (10.07.2019).

Franke, Ulrike y Varma, T. (2019). Independence play: Europe's pursuit of strategic autonomy. European Council on Foreign Relations. Recuperado de: https://www.ecfr.eu/page/-/ECFR_Independence_play_Europe_pursuit_ strategic_autonomy.pdf (I5.09.2019).

Gobierno de Reino Unido (2017). Foreign policy, defence and development.A future partnership paper. Recuperado de: https://assets.publishing.service.gov.uk/government/uploads/system/uploads/attachment_data/file/643924/ Foreign_policy_defence_and_development_paper.pdf 
Grevi, G. (2019). Strategic autonomy for European choices: the key to Europe's shaping power. Discussion paper. European Policy Center. Recuperado de: http://aei.pitt.edu/I00408/I/pub_9300_strategic_autonomy_for_ european_choices2.pdf (15.09.2019).

Grieger, G. (2018). China, the 16+I format and the EU. European Parliamentary Research Service, September 2018. Recuperado de: https://www.europarl.europa.eu/RegData/etudes/BRIE/2018/625I73/EPRS_ BRI(20I8)625I73_EN.pdf (20.04.2020).

Guinea, M. (20/8). La política de Defensa Europea: el avance continúa. Newsletter del Consejo Federal Español del Movimiento Europeo, I I. Recuperado de: http://www.movimientoeuropeo.org/numero-I I/ (I0.09.2019).

Howorth, J. (2018). EU-NATO Cooperation and Strategic Autonomy: logical contradiction or Ariadne's Thread? Working Paper KFG The Transformative Power of Europe, 90. Recuperado de: https://www.polsoz.fu-berlin.de/ en/v/transformeurope/publications/working_paper/wp/WP_90_Howorth/WP_90_Howorth_WEB.pdf $(20.10 .2019)$

Jaguaribe, H. (1979). Hegemonía céntrica y autonomía periférica. Estudios Internacionales, I2 (46), 9|-I80.

Kempin, R. and Kunz, B. (2017). France, Germany and the Quest for European Strategic Autonomy. Notes du Cerfa, |4I. Recuperado de: https://www.ifri.org/sites/default/files/atoms/files/ndc_I4I_kempin_kunz_france_germany_european_strategic_autonomy_dec_2017.pdf (20.10.2019).

Kramp-Karrenbauer, A. (09.03.2019). Acertar en la construcción de Europa. Recuperado en https://www.cdu.de/artikel/ acertar-en-la-construccion-de-europa-getting-europe-right (I0.07.20 I9).

Krotz, U. and Maher, R. (201 I). International Relations theory and the rise of European foreign and security policy. World Politics, 3 (63), 548-579. Recuperado de: https://www.jstor.org/stable/230 I8780?seq=I ( I I.04.2020).

Lawerence, T., Praks, H. y Järvenpää, P. (2017). Building Capacity for the EU Global Strategy. International Centre for Defence and Security. Policy Paper. Recuperado de: https://icds.ee/wp-content/uploads/20I8/ICDS_Policy_Paper_Building_Capacity_for_the_EU_Global_Strategy.pdf (20.10.2019).

Leonard, M. (2019). Inside Macron's Russia Initiative. The Strategist. Recuperado de: https://www.aspistrategist.org.au/ inside-macrons-russia-initiative/ (07.06.2020)

Martinsen, P. M. (septiembre, 2003). The European Security and Defence Strategy: a Strategic Culture in the Making. Trabajo presentado en la Conferencia del Consejo Europeo de Relaciones Exteriores, Marburg.

Meyer, C. O. (2004). Theorising European strategic culture between convergence and the persistence of national diversity. CEPS Working Document, 204. Recuperado de: https://www.ceps.eu/ceps-publications/theorising-european-strategic-culture-between-convergence-and-persistence-national/ (07.06.2020).

Meyer, C. O. (2007). The Quest for a European Strategic Culture:Changing Norms on Security and Defence in the European Union. Basingstoke: Palgrave Macmillan.

Milosevich-Juaristi, M. (20I5). ¿Por qué Rusia es una amenaza existencial para Europa? Real Instituto Elcano,ARI 35/20I5. Recuperado de: http://www.realinstitutoelcano.org/wps/portal/rielcano es/contenido?WCM GLOBAL CONTEXT=/elcano/elcano_es/zonas_es/defensa+y+seguridad/ari35-2015-milosevichjuaristi-rusia-amenaza-existencial-europa (25.11.2019)

Moya, L. E. (2019). The European Intervention Initiative, Permanent Structured Cooperation and French institutional engineering. Documento de Opinión, 79.

Parlamento Europeo (07.10.2019). Hearing with High Representative/Vice President-designate Josep Borrell.

Recuperado de: https://www.europarl.europa.eu/news/en/press-room/20190926IPR62260/hearing-with-highrepresentative-vice-president-designate-josep-borrell (I 4.04.2020).

Puig, J. C. (1980). Doctrinas internacionales y autonomía latinoamericana. Caracas: Instituto de Altos Estudios de América Latina en la Universidad Simón Bolívar.

República Francesa (2017). Revue Stratégique de défense et de securité nationale 2017. Recuperado de: https://www.viepublique.fr/sites/default/files/rapport/pdf/I74000744.pdf (I2.II.2019).

Round, P., Giegerich, B. y Mölling, C. (20I8). European strategic autonomy and Brexit. The International Institute for Strategic Studies.

Schüt, T. and Mölling, C. (2018). Fostering a defence industrial base for Europe: the impact of Brexit. Institute for Strategic Studies.

Simonoff, A. y Lorenzini, M. E. (2019). Autonomía e Integración en las Teorías del Sur: Desentrañando el Pensamiento de Hélio Jaguaribe y Juan Carlos Puig. Iberoamericana - Nordic Journal of Latin American and Caribbean Studies, 48 (I), 96- 106.

Smith, M. (2004). Europe's Foreign and Security Policy: The Institutionalization of Cooperation. New York: Cambridge University Press.

Soler i Lecha, E. y Tocci, N. (2016). Implicaciones de la Estrategia global de la UE para Oriente Medio y el Norte de África. Menara Project, I.

Techau,J.(2016). The EU's New Global Strategy:Useful or Pointless? Carnegie Europe. Recuperado de:https://carnegieeurope. eu/strategiceurope/?fa=63994 (05.1 I.2019).

Thibauld, M. (06.I I.20 I8) Macron pour une «vraie armée européenne»: un projet réalisable? Europe I. Recuperado de: https://www.europe I.fr/politique/macron-pour-une-vraie-armee-europeenne-un-projet-realisable-379483 I (I0.07.2019).

Tokatlian, J.G. y Carvajal, L. (1995). Autonomía y política exterior: un debate abierto, un futuro incierto. Afers Internacionals, 28, 7-3I. Recuperado de: file://C:/Users/240I3/Downloads/28tokatliancarvajal\%20(2).pdf 
(20.04.2020).

Varga, G. (2017). Towards European Strategic Autonomy? Evaluating the New CSDP Initiatives. Budapeste: Institute for Foreign Affairs and Trade. Recuperado de: https://kki.hu/assets/upload/07_KKI-Studies_CSDP_VargaG_20171003. pdf (07.06.2020).

Vilmer J., Escorcia, A., Guillaume, M. y Herrera, J. (2018). Information Manipulation: A Challenge for our Democracies. Resource Centre on Media Freedom in Europe. Recuperado de: https://www.rcmediafreedom.eu/Publications/Reports/Information-Manipulation-A-Challenge-for-Our-Democracies (05.04.2020). 


\section{RELACIONES INTERNACIONALES}

Revista académica cuatrimestral de publicación electrónica Grupo de Estudios de Relaciones Internacionales (GERI)

Universidad Autónoma de Madrid, España

https://revistas.uam.es/relacionesinternacionales

ISSN 1699 - 3950

f facebook.com/RelacionesInternacionales

3. twitter.com/RRInternacional

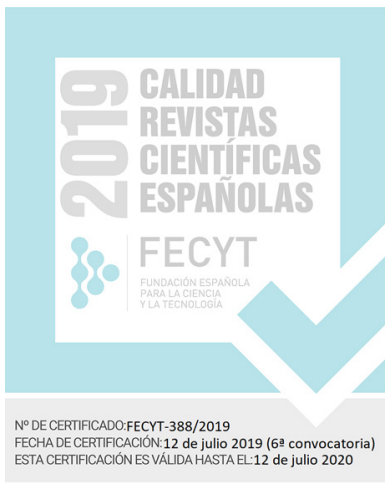

